\title{
Mixed crystalline films of co-evaporated hydrogen- and fluorine-terminated phthalocyanines and their application in photovoltaic devices
}

\author{
Andreas Opitz ${ }^{\mathrm{a}}$, Bernhard Ecker ${ }^{\mathrm{a}}$, Julia Wagner ${ }^{\mathrm{a}}$, Alexander Hinderhofer ${ }^{\mathrm{b}}$, \\ Frank Schreiber ${ }^{\mathrm{b}}$, Jochen Manara ${ }^{\mathrm{c}}$, Jens Pflaum ${ }^{\mathrm{c}}$, Wolfgang Brütting ${ }^{\mathrm{a}}$ \\ ${ }^{a}$ Institute of Physics, University of Augsburg, Augsburg, Germany \\ ${ }^{b}$ Institute of Applied Physics, University of Tübingen, Tübingen, Germany \\ ${ }^{c}$ Institute for Experimental Physics VI, University of Würzburg, and Bavarian Center for Applied Energy Research, 97074 Würzburg, Germany
}

\begin{abstract}
Blends of organic electron and hole conductive materials are widely used for ambipolar charge carrier transport and photovoltaic cells. An obvious choice for donor-acceptor blends are organic semiconducting materials in their hydrogenated and fluorinated form, since they combine potentially suitable electronic properties with structural compatibility of the two constituents. This study focuses on the structural, optical, and electrical properties of blends using hydrogenated copper-phthalocyanine $\left(\mathrm{H}_{16} \mathrm{CuPc}\right)$ in combination with its perfluorinated version $\left(\mathrm{F}_{16} \mathrm{CuPc}\right)$. Using X-ray scattering, scanning force microscopy and optical absorption measurements we show that mixed crystalline films are obtained by co-evaporation of the two materials. Electrical transport measurements reveal a profound reduction of the current for bipolar charge injection in mixed films. We discuss the formation of self-trapped charge-transfer excitons as possible explanation for this unexpected behaviour, which impedes the usability of this system in photovoltaic cells.
\end{abstract}

Key words:

phthalocyanines, vacuum deposition, mixed crystalline films, photovoltaic cells

\section{Introduction}

Phthalocyanines are well-known metal-organic complexes for several decades [1]. They have been used for Schottkybarrier solar cells [2] and as hole transporting layers in organic light-emitting diodes [3]. More recently, they are used as absorber and electron donor in organic photovoltaic cells in combination with strong electron acceptors, such as the fullerene $\mathrm{C}_{60}[4,5,6,7,8]$. In this context, modification of the molecular energy levels, viz. the ionization potential (IP) or highest occupied molecular orbital (HOMO) as well as the electron affinity (EA) or lowest unoccupied molecular orbital (LUMO), by changing the termination of the outer phenyl rings has become an interesting tool to tune electronic properties leaving the optical properties almost unchanged [9]. With increasing number of fluorine atoms bound to the outer phenyl ring the IP and EA shift away from the vacuum level $[9,10]$. As an example the IP measured by ultra-violet photoelectron spectroscopy shifts from $5.0 \mathrm{eV}$ for hydrogenated copper-phthalocyanine $\left(\mathrm{H}_{16} \mathrm{CuPc}\right)$ to $6.1 \mathrm{eV}$ for its per-fluorinated version $\left(\mathrm{F}_{16} \mathrm{CuPc}\right)$ [10], so that the combination of both materials could be of interest for donor-acceptor (DA) systems with comparable sizes of the molecules and the $\pi$-conjugated systems. Together with a similar crystal structure (see later) this gives rise to the expectation that mixed crystalline films by co-evaporation of the

Email addresses: Andreas.Opitz@physik.uni-augsburg.de (Andreas Opitz), Wolfgang.Bruetting@physik.uni-augsburg.de (Wolfgang Brütting) two materials can be obtained for applications in organic photovoltaics.

The combination of $\mathrm{H}_{16} \mathrm{CuPc}$ and $\mathrm{F}_{16} \mathrm{CuPc}$ was used before in bilayered field-effect transistors (FET) $[11,12,13]$ to obtain ambipolar transport. For both materials a threshold voltage shift was observed if the respective other material is deposited on top of the transport material in the FET. For thicker layers the second deposited material opens an additional channel for the opposite charge carrier type to give ambipolar transport. The threshold voltage shift was related to a charge transfer with holes accumulating in $\mathrm{H}_{16} \mathrm{CuPc}$ and electrons in $\mathrm{F}_{16} \mathrm{CuPc}$. Therefore the bilayer structure can be used to engineer the threshold voltage in FETs.

Mixed crystals of strong DA systems like TTF-TCNQ are well-known since the 1970s [14]. However, the growth of crystalline thin films of two organic semiconductors by vacuum deposition is a relatively new aspect for organic electronics. It has been demonstrated that charge transfer salts, such as TTF-TCNQ and its many derivatives, can be deposited as crystalline films by thermal evaporation of the bulk material [15]. The formation of mixed crystalline films by coevaporation of molecules has only recently been observed for rod-like molecules [16], such as sexithiophene (6T), sexiphenyl (6P) and dihexylsexithiophene (DH6T). Thereby the structure of the films is predominantly determined by the size of the conjugated system. It was also shown that pentacene and perfluoropentacene can be grown as intercalated mixed crystalline layers on top of $\mathrm{SiO}_{2}$; thereby the ionization energy can be continu- 
ously varied by the layer composition [17].

Here we present a study of blends using hydrogenated copper-phthalocyanine $\mathrm{H}_{16} \mathrm{CuPc}$ in combination with its perfluorinated version $\mathrm{F}_{16} \mathrm{CuPc}$. In addition to studying the fundamental structural, optical, and electrical properties centering around the question of the formation of mixed crystals we investigate the usability of these materials for photovoltaic cells as planar and bulk-heterojunction devices.

\section{Experimental}

The materials used in this study were purchased from Sigma Aldrich as sublimation grade and additionally purified by temperature gradient sublimation; for $\mathrm{F}_{16} \mathrm{CuPc}$ the sublimation procedure was repeated once. The structural formulae are given in fig. 1. The organic semiconductor films were deposited by thermal evaporation from two independent low-temperature effusion cells in a vacuum better than $10^{-7}$ mbar. The thickness of the films was controlled via deposition monitors using quartz microbalances. For mixed films two independent monitors were used. The layer sequence for the bilayer structure consists of $\mathrm{H}_{16} \mathrm{CuPc}$ deposited on the hole injecting layer followed by $\mathrm{F}_{16} \mathrm{CuPc}$. The deposition rates were $0.35 \AA / \mathrm{s}$ for neat films and up to $1.4 \AA / \mathrm{s}$ for the material with the higher volume fraction in the mixtures. As substrates glass slides covered with $130 \mathrm{~nm}$ of indium-tin oxide (ITO) and $30 \mathrm{~nm}$ of the conducting polymer polyethylenedioxythiophene-polystyrenesulfonate (PEDOT:PSS, purchased from H.C. Starck as BAYTRON P) were used. For the optical absorption measurements the films were deposited on quartz glass slides. During the deposition of the phthalocyanine molecules the substrate was held either at room temperature (about $300 \mathrm{~K}$ ) or at an elevated temperature (about $375 \mathrm{~K}$ ).

The charge transport properties were analyzed in both unipolar and ambipolar diodes. For hole-only diodes the active organic layer was deposited on an ITO/PEDOT:PSS anode and a $100 \mathrm{~nm}$ thick $\mathrm{Au}$ film with a thin interlayer of 2,3,5,6tetrafluoro-7,7,8,8-tetracyano-quinodimethane ( $\left.\mathrm{F}_{4} \mathrm{TCNQ}\right)$ was used as hole injecting layer [18]. The electron-only diodes contained a $100 \mathrm{~nm}$ thick Al bottom electrode and a $100 \mathrm{~nm}$ thick $\mathrm{Al}$ electrode on the top of the organic film with a $0.5 \mathrm{~nm}$ thick interface doping layer of $\mathrm{LiF}$ for the latter one. Using an ITO/PEDOT:PSS bottom contact together with a LiF/A1 top electrode ambipolar injection and charge transport was obtained. The active organic layer in all investigated devices consisted of an $80 \mathrm{~nm}$ thick film of neat or blended materials (volume mixing ratio of $1: 1$ ). The active area was about $2 \times 2 \mathrm{~mm}^{2}$. Electrical characterization was performed without air-exposure under vacuum or in a nitrogen filled glove box.

To analyze the current-voltage characteristics the measured curves were fitted by the model of trap-free space charge limited currents (SCLC, eq. 1) [19] combined with a Poole-Frenkel type field-dependent mobility [20], which gives the current density as

$$
j_{\mathrm{SCLC}}=\frac{9}{8} \cdot \mu_{0} \cdot \varepsilon_{0} \varepsilon_{\mathrm{r}} \frac{V_{\mathrm{eff}}^{2}}{d^{3}} \exp \left[\gamma \sqrt{\frac{V_{\mathrm{eff}}}{d}}\right] .
$$
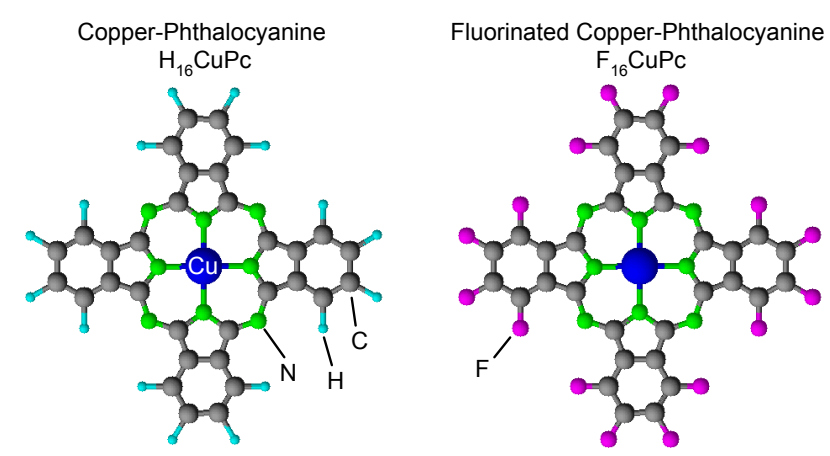

Figure 1: Structural formulae of hydrogen-terminated copper phthalocyanine $\mathrm{H}_{16} \mathrm{CuPc}$ and fluorine-terminated copper phthalocyanine $\mathrm{F}_{16} \mathrm{CuPc}$.

This equation contains a zero-field mobility $\mu_{0}$ and a field activation parameter $\gamma . d$ is the film thickness and $V_{\text {eff }}$ the effective applied voltage $V-V_{\mathrm{Bi}}$, with $V_{\mathrm{Bi}}$ being the built-in voltage, i.e. the difference of the injection barriers at both electrodes. The parameters $\mu_{0}, \gamma$ and $V_{\mathrm{Bi}}$ are determined by fitting the measured data in the higher voltage range above $V_{\mathrm{Bi}}$.

Devices showing ambipolar injection and transport, i.e. devices comprising ITO/PEDOT:PSS and $\mathrm{LiF} / \mathrm{Al}$ electrodes were additionally investigated as solar cells. In this case the active organic film consisted either of an $80 \mathrm{~nm}$ thick 1:1 blend or two neat layers with $40 \mathrm{~nm}$ thickness for each material. Currentvoltage characteristics of the solar cells were measured in darkness and under illumination. The intensity of the solar simulator (AM1.5 filters) was varied up to $100 \mathrm{~mW} / \mathrm{cm}^{2}$, i.e. one sun.

In addition to the electrical measurements, the neat and blended organic films were analyzed by scanning force microscopy (SFM) and specular X-ray reflectometry. The SFM measurements were performed using a Thermo Microscopes Autoprobe CP-Research in non-contact mode. The X-ray scattering measurements were conducted on a GE/Seifert $\mathrm{x}$-ray diffractometer $(\mathrm{Cu} \mathrm{K} \alpha 1$ radiation, multilayer mirror, and double bounce compressor monochromator). The optical absorption spectra were recorded for films deposited on quartz glass substrates using a Perkin Elmer Lambda 950 with a spectral range from 0.5 to $5.0 \mathrm{eV}$. X-ray scattering, SFM, and optical absorption measurements were performed under ambient conditions.

\section{Results and discussion}

\subsection{Structural properties}

The morphology of phthalocyanine films deposited on PEDOT:PSS/ITO was determined by non-contact scanning force microscopy. The results are shown in fig. 2. Neat films evaporated at room temperature have a granular structure with a typical grain size of about $50 \mathrm{~nm}$. Phthalocyanine films deposited at elevated temperature display a needle-like structure with a typical length of about $300 \mathrm{~nm}$. The higher temperature during evaporation allows a faster diffusion of the molecules and thereby a better ordering resulting in these needle-like crystals. Blended phthalocyanine films show a similar morphology and roughness as the neat films at the same deposition conditions. 

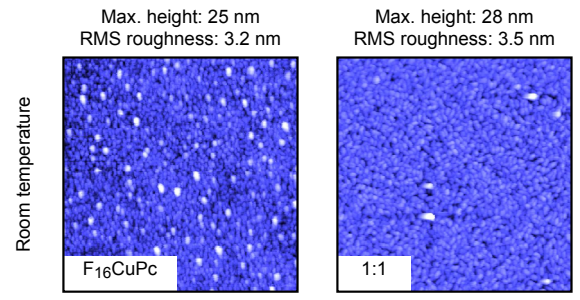

Max. height: $15 \mathrm{~nm}$ RMS roughness: $2.1 \mathrm{~nm}$

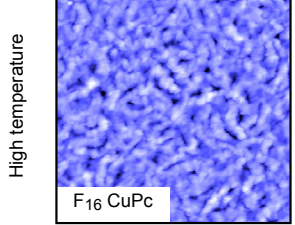

Max. height: $15 \mathrm{~nm}$ RMS roughness: $2.0 \mathrm{~nm}$

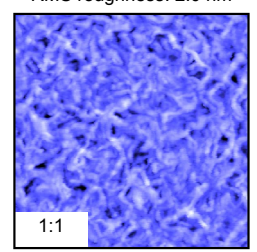

Max. height: $49 \mathrm{~nm}$

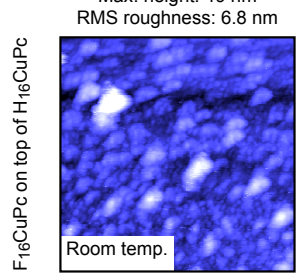

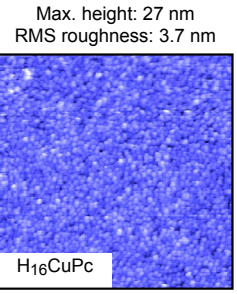

Max. height: $15 \mathrm{~nm}$ RMS roughness: $2.0 \mathrm{~nm}$

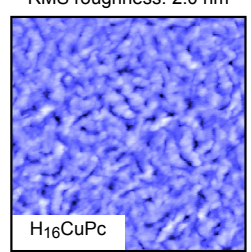

$2 \times 2 \mu m^{2}$

Max.

$0 \mathrm{~nm}$

Figure 2: Scanning force microscopy images taken in non-contact mode for neat $\mathrm{F}_{16} \mathrm{CuPc}$ and $\mathrm{H}_{16} \mathrm{CuPc}$ films as well as for a 1:1 blended film grown at $300 \mathrm{~K}$ (upper row) and $375 \mathrm{~K}$ (middle row) on PEDOT:PSS films. In the lower row the surface morphology of $\mathrm{F}_{16} \mathrm{CuPc}$ films deposited on top of a $\mathrm{H}_{16} \mathrm{CuPc}$ film is shown for deposition at room and high temperature, respectively. The total image size is $2 \times 2 \mu \mathrm{m}^{2}$. The max. height is given as the difference between the lowest value (dark blue) and the highest value (white) in each of the images.

This similarity in morphology is a first indication for the formation of mixed crystalline films. To confirm this, the films were further analyzed by X-ray scattering.

Figure 3 shows the out-of-plane first order diffraction peak for the neat films as well as for the 1:1 blend deposited at room temperature. Importantly, the blended $\mathrm{H}_{16} \mathrm{CuPc} / \mathrm{F}_{16} \mathrm{CuPc}$ film shows only one diffraction peak located between the diffraction peaks of the neat materials. The peak width is similar to that of the neat films and the lattice spacing lies between those of the neat materials, consistent with a truly mixed crystalline film, which was already observed for 1:1 mixtures of different hydrogen terminated planar phthalocyanines [21]. The dependence of the lattice spacing on the concentration is summarized in fig. 3 (right). Additional blend ratios are included (3:1 and 1:3) which were not presented in fig. 3 (left) for clarity reasons. The lattice parameter for the mixed crystal system $\mathrm{H}_{16} \mathrm{CuPc} / \mathrm{F}_{16} \mathrm{CuPc}$ changes linearly with the concentration between the lattice parameters of the neat materials. This linear change is related to the gradual change of the content of the two planar molecular species with different diameter in the blends. The more crystalline films deposited at higher substrate temperature show basically the same lattice spacing with a slightly reduced peak width related to larger crystallites with coherent diffraction in agreement with the SFM analysis.

At this point it is useful to compare with other structural data.
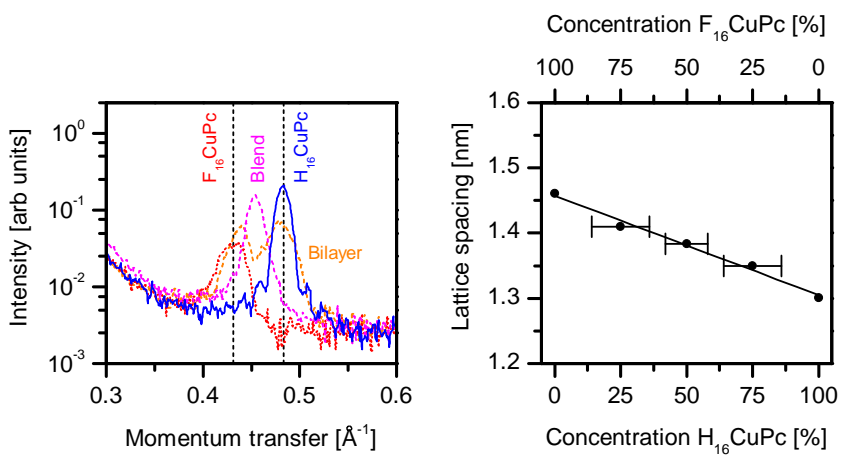

Figure 3: (left) X-ray diffraction spectra of neat films, a 1:1 blend and a bilayer structure evaporated onto PEDOT:PSS at room temperature. (right) Analysis of the lattice spacing determined from X-ray scattering spectra of films with different mixing ratios. The straight line is the linear fit of the measurement points.

Identified by the Bragg-reflection at $q_{\mathrm{z}}=0.48 \AA^{-1}$, neat thin films of $\mathrm{H}_{16} \mathrm{CuPc}$ on PEDOT:PSS crystallize in the $\alpha$-phase, which is common for $\mathrm{H}_{16} \mathrm{CuPc}$ thin film growth [22]. This was earlier determined to be isostructural with other phthalocyanine $\alpha$-phases, which show a herringbone structure with two molecules per unit cell [23]. However a later redetermination has shown that the $\mathrm{H}_{16} \mathrm{CuPc} \alpha$-phase has a triclinic structure with only one molecule per unit cell [24]. According to this structural data the peak at $q_{\mathrm{z}}=0.48 \AA^{-1}$ corresponds to the (100) reflection, which means that the $\mathrm{H}_{16} \mathrm{CuPc}$ molecules are standing nearly upright and stack in the direction parallel to the substrate. For growth on $\mathrm{SiO}_{2} \mathrm{~F}_{16} \mathrm{CuPc}$ thin films exhibit two polymorphs coined $\beta$-phase and $\beta_{\text {bilayer-phase }[25,26] \text {. The }}$ two polymorphs exhibit different in-plane-stacking behaviour, in particular the $\beta$-phase has a herringbone arrangement, where the $\beta_{\text {bilayer-phase has a single molecule per unit cell and builds }}$ stacks parallel to the substrate similar to the $\mathrm{H}_{16} \mathrm{CuPc} \alpha$-phase. Since both $\mathrm{F}_{16} \mathrm{CuPc}$ polymorphs have an identical out-of-plane spacing, it cannot be determined by the data of fig. 3 whether a herringbone arrangement is present in the neat film or not.

Due to the different size of the terminating atoms the size of the molecule varies and thereby also the lattice spacing $a$ perpendicular to the substrate (see fig. 4) [24, 26]. The values are about $1.24 \mathrm{~nm}$ for the $\alpha$-phase of $\mathrm{H}_{16} \mathrm{CuPc}$ and about $1.43 \mathrm{~nm}$ for $\mathrm{F}_{16} \mathrm{CuPc}$ films, respectively. Several other molecular arrangements have been observed. E.g. $\mathrm{H}_{16} \mathrm{CuPc}$ crystallizes in a $\beta$-phase when heated higher than $480 \mathrm{~K}$ [27]. For the present study the key point is not the subtle differences between different polymorphs, but rather the fact that the ordering motifs for both compounds are similar, thus facilitating the formation of mixed crystalline films. Figure 4 shows schematic film structures for neat phthalocyanines and a 1:1 mixture. A gradual change of the layer spacing can be rationalized by a varying content of the two molecular species with different size and a change of the tilt angle.

We note that the observation of a single diffraction peak in the blends does not automatically imply the formation of an ordered mixed crystalline film with an in-plane superstructure (as 
fig. 4 would suggest). It merely indicates that both constituents are homogenously mixed on a molecular length scale without detectable phase separation or precipitation.

The bilayer system containing a neat $\mathrm{H}_{16} \mathrm{CuPc}$ and a neat $\mathrm{F}_{16} \mathrm{CuPc}$ film was analyzed, too. The SFM images are shown in fig. 2 (third row). The $\mathrm{F}_{16} \mathrm{CuPc}$ film on top of the $\mathrm{H}_{16} \mathrm{CuPc}$ film evaporated at room temperature is full of fissures. When the evaporation is performed at elevated temperature the film is closed and displays needle-like crystals. The room temperature grown film already shows XRD peaks (see fig. 3), but only films evaporated at elevated temperatures have closed layers and can be used for bilayer devices.

\subsection{Optical properties}

Figure 5 shows the optical absorption under normal incidence for neat phthalocyanine films as well as a blended and a bilayer film. Both materials are almost transparent in the range from 2.5 to $3.0 \mathrm{eV}$. The absorption of $\mathrm{H}_{16} \mathrm{CuPc}$ films between 1.4 and $2.3 \mathrm{eV}$ is related to the Q-band and the absorption in the UV-range (between 3.1 and $4.0 \mathrm{eV}$ ) to the B-band [28]. The Q-band absorption of $\mathrm{H}_{16} \mathrm{CuPc}$ shows one strong peak at about $1.81 \mathrm{eV}$ in solution and displays two strong peaks in the solid state (peaks at $1.80 \mathrm{eV}$ and $1.97 \mathrm{eV}$ ). As mentioned before $\mathrm{F}_{16} \mathrm{CuPc}$ films crystallize in two different crystal structures $[25,26]$ with different absorption behaviour. In the visible range one structure is related to the peaks at $1.89 \mathrm{eV}$ and $1.77 \mathrm{eV}$ and the other structure has a large crystal shift resulting in a peak around $1.56 \mathrm{eV}$ [29].

The measured spectrum of the blend (Figure 5, left) shows one strong peak at $1.97 \mathrm{eV}$ and a shoulder at about $1.80 \mathrm{eV}$. These features are located in the spectral range were both molecules show absorption. In addition, the far red-shifted peak at $1.56 \mathrm{eV}$ in $\mathrm{F}_{16} \mathrm{CuPc}$ disappears indicating that the related packing motif is no longer present in the blend. This is another demonstration of the formation of mixed crystalline films by co-evaporation of the two different phthalocyanine molecules.

The absorption of a bilayered film $\left(\mathrm{F}_{16} \mathrm{CuPc}\right.$ on top of a $\mathrm{H}_{16} \mathrm{CuPc}$ film) is shown in fig. 5 (right). There all peaks of the neat films are present and scale with the doubled film thickness

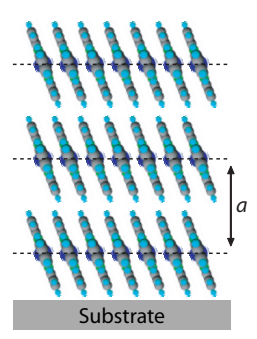

$\mathrm{H}_{16} \mathrm{CuPc}$

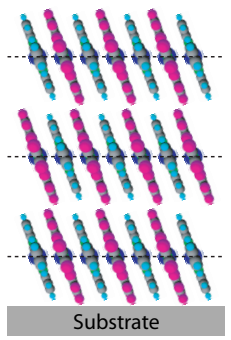

$\mathrm{H}_{16} \mathrm{CuPc}: \mathrm{F}_{16} \mathrm{CuPc}$ $1: 1$

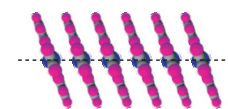
Hnt IIII

$\mathrm{F}_{16} \mathrm{CuPc}$
Figure 4: Schematic molecular arrangement for neat and mixed phthalocyanine films. The interlayer spacing changes gradually from $\mathrm{H}_{16} \mathrm{CuPc}$ to the blend and further to $\mathrm{F}_{16} \mathrm{CuPc}$. For easier visualization an ordered structure of the blend is assumed. The lattice parameter $a$ is about $1.3 \mathrm{~nm}$ related to the $\alpha$-phase in $\mathrm{H}_{16} \mathrm{CuPc}$ and about $1.45 \mathrm{~nm}$ for $\mathrm{F}_{16} \mathrm{CuPc}[24,26]$.
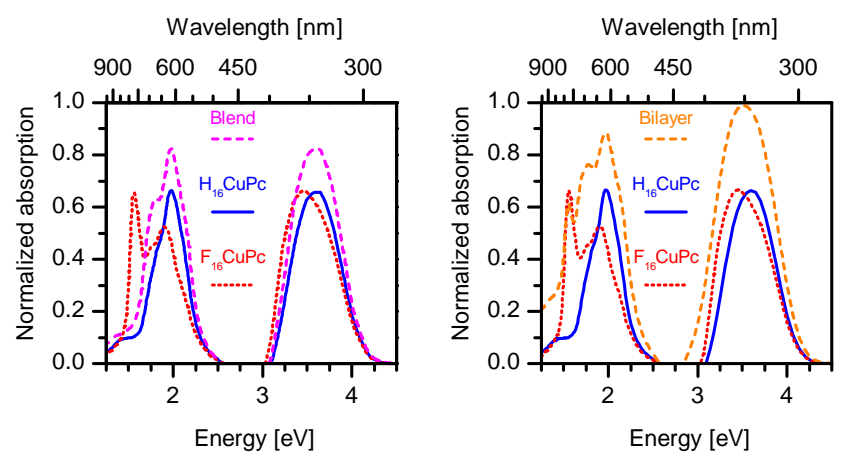

Figure 5: Absorption spectra of neat as well as blended (left) and bilayered (right) films using $\mathrm{H}_{16} \mathrm{CuPc}$ and $\mathrm{F}_{16} \mathrm{CuPc}$ deposited on quartz glass substrates at room temperature. The bilayer structure consists of an $\mathrm{H}_{16} \mathrm{CuPc}$ film on top of an $\mathrm{F}_{16} \mathrm{CuPc}$ film which shows the similar behaviour as the opposite film sequence. Additionally a higher on-set at the low energy side of the spectrum is observed for the bilayer structure as for the other samples which is related to differences in the interference effect due the larger film thickness.

in the bilayered system (40 $\left.\mathrm{nm} \mathrm{H}_{16} \mathrm{CuPc}+40 \mathrm{~nm} \mathrm{~F}_{16} \mathrm{CuPc}\right)$ as compared to the neat films $(40 \mathrm{~nm})$.

\subsection{Electrical properties}

The current-voltage characteristics were measured using various electrodes to realize hole-only, electron-only and ambipolar transport [30] and are shown in fig. 6. In all samples unipolar electron currents are higher than unipolar hole currents. For the ambipolar transport, however, there is a huge difference between neat materials and the 1:1 blend. While for the former the ambipolar current is close to the current in the electron-only devices, indicating that the current is carried mostly by electrons, the latter system shows a reduction of the current by about four orders of magnitude in comparison to the ambipolar currents of the neat films and both unipolar currents in the blend. The resulting mobilities using the before described SCLC model are also shown in fig. 6 .

The unipolar mobilities of the blend are located between the unipolar mobilities of the neat materials. This alignment of the unipolar mobilities shows similar $\pi$ - $\pi$-overlap in the mixed crystalline films as in the neat films. However, the ambipolar mobility in the blended film is orders of magnitude lower than both unipolar mobilities and the ambipolar mobilities of the neat materials. The same trend is observed for films grown on room temperature substrates, though, the reduction of the ambipolar mobility is more pronounced in the heated films. As the unipolar mobilities in the 1:1 mixture are just in between the values for neat films, the ambipolar transport cannot be limited by the absence of percolation pathways. The strongly reduced ambipolar mobility should rather be related to the simultaneous presence of both charge carrier types in the mixture. A possible explanation is the formation of charge transfer (CT) excitons by the injected charge carriers. These CT states will then limit the transport by blocking the occupied molecules for further injected charge carriers; they would be self-trapped. The idea of generation of charge transfer excitons is supported by the high EA of $\mathrm{F}_{16} \mathrm{CuPc}$. As mentioned in the introduction, the IP is 

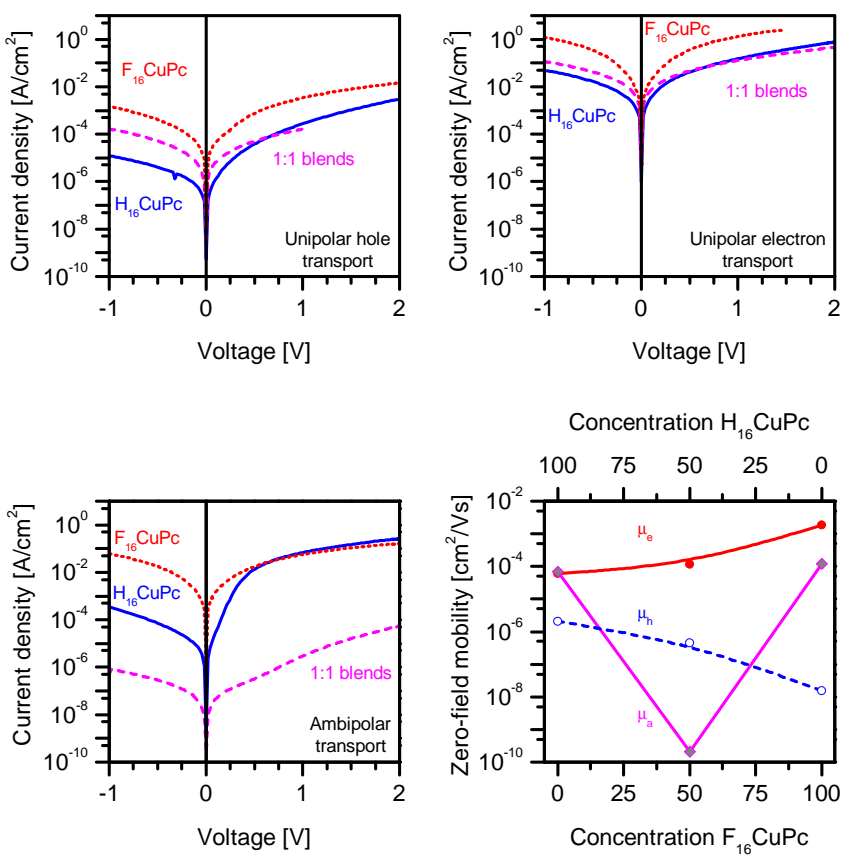

Figure 6: Current-voltage characteristics of electron-only, hole-only and ambipolar diodes with neat $\mathrm{H}_{16} \mathrm{CuPc}$ and $\mathrm{F}_{16} \mathrm{CuPc}$ as well as a 1:1 blend as active layer. All films were deposited using high substrate temperatures. The lower right diagram shows the zero-field mobilities determined using an SCLC model for all nine diodes.

$5.0 \mathrm{eV}$ for $\mathrm{H}_{16} \mathrm{CuPc}$ and $6.1 \mathrm{eV}$ for $\mathrm{F}_{16} \mathrm{CuPc}$, respectively. Taking identical transport gaps of $2.3 \mathrm{eV}$ for both materials [31], results in a LUMO level of $\mathrm{F}_{16} \mathrm{CuPc}$ being only $1.2 \mathrm{eV}$ above the HOMO of $\mathrm{H}_{16} \mathrm{CuPc}$. Thus, an intermolecular low-lying CT state could be possible in $\mathrm{H}_{16} \mathrm{CuPc} / \mathrm{F}_{16} \mathrm{CuPc}$ blends. However, as we do not see a signature in the optical absorption measured down to $0.6 \mathrm{eV}$ (not shown here), a direct electronic transition into and from this CT state seems to be not allowed, thus stabilizing the charge separated state. Consistently, due to the ambipolar nature of CT excitons the unipolar mobilities would not be affected.

The possibility of self-trapped CT excitons was suggested before for molecular crystals consisting of stacked alternating donor-acceptor molecules, such as anthracene-PMDA [32]. Materials of this type are insulating and CT excitons created by optical excitation are not mobile. We suggest that this could also happen in the system presented here for simultaneous injection of electrons and holes into a 1:1 blend.

We note that, strictly speaking, differences between electrononly and ambipolar devices could also be caused by different film growth modes, because in the former case $\mathrm{Al}$ is used as substrate whereas the latter films are grown on PEDOT:PSS. However, as there is also a large difference of the mobility in the blend between unipolar hole-only and ambipolar devices, which have the same PEDOT:PSS layer as anode, we do not consider the substrate as the decisive parameter for the significant drop of the ambipolar mobility in the 1:1 blend.
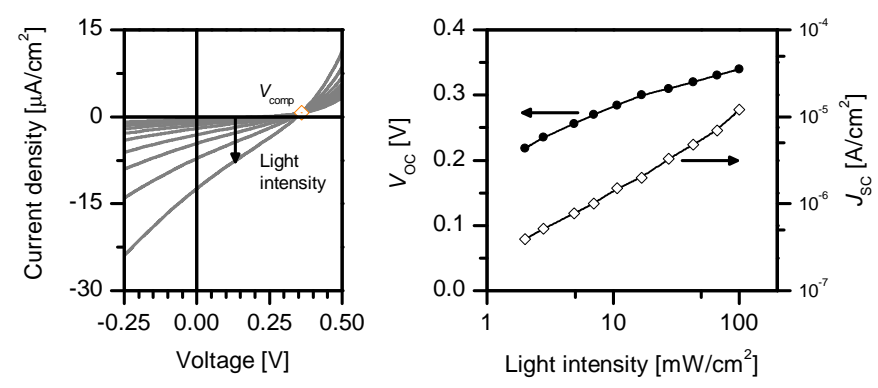

Figure 7: (left) Current-voltage characteristics for the blended $\mathrm{H}_{16} \mathrm{CuPc} / \mathrm{F}_{16} \mathrm{CuPc}$ solar cell with an active layer deposited at high temperature. The curves are shown for different white light intensities (simulated AM1.5 illumination). The compensation voltage $V_{\text {Comp }}$ is determined as the crossing point of all curves [33]. (right) Open circuit voltage ( $\left.V_{\mathrm{OC}}\right)$ and short circuit current density $\left(J_{\mathrm{SC}}\right)$ determined from the left diagram as function of the light intensity.

\subsection{Photovoltaic cells}

Organic donor and acceptor materials are promising candidates for photovoltaic cells [34]. In these cells the generated excitons dissociate into free charge carriers at the donor/acceptor interface by an ultra-fast photo-induced charge transfer [35, 36]. To create this donor/acceptor interface bilayered (planar heterojunction) [4] and blended (bulk-heterojunction) [5, 37] photovoltaic cells are employed. In bilayered systems the dissociation is localized at the interface between the two layers, while in bulk-heterojunction cells it occurs within the whole volume of the blended film. Due to this mixture of donor and acceptor materials the photon-to-current conversion efficiency and the power conversion efficiency were found to increase $[5,37,38]$. An obvious choice for donor-acceptor blends are organic semiconducting materials in their hydrogenated and fluorinated form, since they combine potentially suitable electronic properties with potential structural compatibility of the two constituents.

Figure 7 shows the $I-V$ characteristics for a solar cell containing a blended $\mathrm{H}_{16} \mathrm{CuPc} / \mathrm{F}_{16} \mathrm{CuPc}$ film as active layer deposited at elevated temperature. The measurement includes the dark current and the current under various illumination intensities up to one sun. The compensation voltage is about $0.36 \mathrm{eV}$ [33] and slightly higher than the open circuit voltage at the highest intensity. The short circuit currents are orders of magnitudes lower than comparable cells made from $\mathrm{CuPc} / \mathrm{C}_{60}$ blends [39, 40]. Regarding the low ambipolar mobility in the $\mathrm{H}_{16} \mathrm{CuPc} / \mathrm{F}_{16} \mathrm{CuPc}$ blends the very low photocurrents are probably related to the above mentioned self-trapping process. However, the charge transfer excitons are not formed by a direct optical transition but rather by a photo-induced charge transfer after light absorption by one of the two molecular species. The fill factor of about $25 \%$ and a power conversion efficiency of only $0.002 \%$ shows the ill performance and the limited usability of this system in photovoltaic cells. Comparable solar cells deposited at room temperature show an even smaller open circuit voltage and similar short circuit currents as the heated ones. The power efficiency is reduced to $0.001 \%$. 

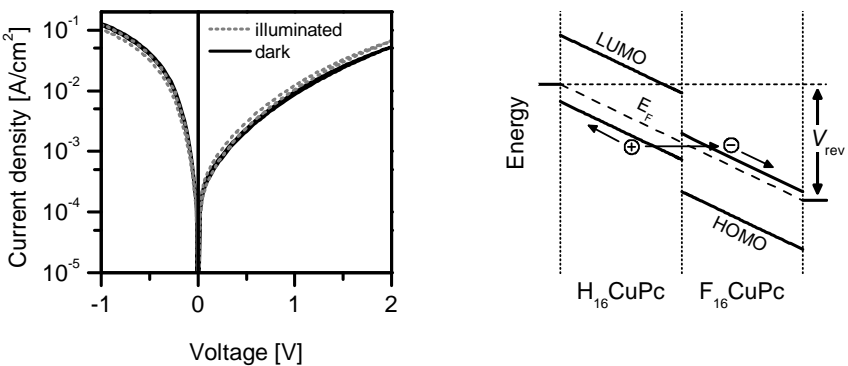

Figure 8: (left) Current-voltage characteristic for an ambipolar $\mathrm{H}_{16} \mathrm{CuPc} / \mathrm{F}_{16} \mathrm{CuPc}$ bilayer diode in the dark and under one sun illumination. (right) Energy level diagram for an $\mathrm{H}_{16} \mathrm{CuPc} / \mathrm{F}_{16} \mathrm{CuPc}$ bilayer diode to explain the charge generation layer under reverse bias conditions. The energy levels for $\mathrm{H}_{16} \mathrm{CuPc}$ are taken from our own measurements and the energy levels for $\mathrm{F}_{16} \mathrm{CuPc}$ from the literature [41].

Fig. 8 (left) shows the $I-V$ characteristics of the planar $\mathrm{H}_{16} \mathrm{CuPc} / \mathrm{F}_{16} \mathrm{CuPc}$ heterojunction in the dark and under white light illumination at one sun. Comparing to fig. 6 one immediately notices a remarkable difference: the dark current at $2 \mathrm{~V}$ is about 6 orders of magnitude higher than in the blend, however, there is almost no effect of illumination. Even more remarkable is the fact that the reverse current at $-1 \mathrm{~V}$ is about one order of magnitude higher than the forward current at $+1 \mathrm{~V}$. Forward biasing allows for the injection of electrons into $\mathrm{F}_{16} \mathrm{CuPc}$ and holes into $\mathrm{H}_{16} \mathrm{CuPc}$. Both carrier species are transported to the interface between both materials where they recombine. The formation of CT excitons is not an issue in this case due to the applied electric field across the interface. Under reverse bias conditions the injection of charge carriers should be blocked by large energy barriers at the electrodes. However, if the energy offset between the HOMO of $\mathrm{H}_{16} \mathrm{CuPc}$ and the LUMO of $\mathrm{F}_{16} \mathrm{CuPc}$ is small enough, tunneling of electrons from the further into the latter should be possible as shown in fig. 8 (right). This effect of creating an electron-hole pair under reverse bias conditions at an interface between a p- and an n-type material is known as charge generation layer and has been employed in multilayer stacked organic LEDs and tandem solar cells $[42,43]$. We also investigated the influence of light for the planar heterojunction. As visible in fig. 8 the forward current is only weakly increased by photo-generated charge carriers. In backward direction, however, this photo-induced change is obscured by the higher current due to the charge generation layer. Due to the absence of an open circuit voltage and the weak influence of light, this planar heterojunction can not be used as photoactive interface in organic photovoltaic cells.

\section{Summary}

In conclusion, we have shown that molecularly mixed crystalline films can be grown by co-evaporation of $\mathrm{H}_{16} \mathrm{CuPc}$ and $\mathrm{F}_{16} \mathrm{CuPc}$ on device-relevant ITO/PEDOT:PSS substrates. These blends show the usual X-ray spectrum and needle-like film morphology known from neat films of phthalocyanines. Due to different size of the molecules, a continuous change of the lattice spacing is observed by changing the mixing ratio. Optical absorption measurements confirm this scenario: they are not just a superposition of the spectra of neat materials, as would be expected for phase-separated blends, but show distinct new features. In particular the disappearance of the characteristic band at $1.56 \mathrm{eV}$ in $\mathrm{F}_{16} \mathrm{CuPc}$ is a clear indication of molecular mixing of both species.

A CT exciton could serve as an explanation for the unexpectedly low charge carrier mobility in ambipolar blends, which is found to be orders of magnitude lower than the ambipolar mobility in neat materials and the electron and hole mobilities in unipolar devices. The reduced mobility is also the origin of the poor performance of photovoltaic cells containing $\mathrm{H}_{16} \mathrm{CuPc} / \mathrm{F}_{16} \mathrm{CuPc}$ blends. The charge collection efficiency is drastically reduced by the suggested formation of bound CT excitons. In contrast, a bilayered $\mathrm{H}_{16} \mathrm{CuPc} / \mathrm{F}_{16} \mathrm{CuPc}$ device works as a charge generation layer which could be of interest in tandem solar cells.

\section{Acknowledgement}

This work was supported by the Deutsche Forschungsgemeinschaft under Priority Programme 1355 "Elementary Processes of Organic Photovoltaics".

\section{References}

[1] C. C. Lenzoff, A. B. P. Lever, ed., Phthalocyanines, VCH, New York, 1996.

[2] J. Simon, J.-J. André, Molecular Semiconductors, Springer, Berlin, 1984.

[3] S. A. van Slyke, C. H. Chen, C. W. Tang, Organic electroluminescent devices with improved stability, Appl. Phys. Lett 69 (1996) 2160-2162.

[4] C. W. Tang, Two-layer organic photovoltaic cell, Appl. Phys. Lett. 48 (1986) 183-185.

[5] J. Rostalski, D. Meissner, Monochromatic versus solar efficiencies of organic solar cells, Sol. Energy Mater. Sol. C. 61 (2000) 87-95.

[6] T. Stübinger, W. Brütting, Exciton diffusion and optical interference in organic donor-acceptor photovoltaic cells, J. Appl. Phys. 90 (2001) 36323641.

[7] P. Peumans, A. Yakimov, S. R. Forrest, Small molecular weight organic thin-film photodetectors and solar cells, J. Appl. Phys. 93 (2003) 36933723.

[8] M. Riede, T. Mueller, W. Tress, R. Schueppel, K. Leo, Small-molecule solar cells - status and perspectives, Nanotechnology 19 (2008) 424001.

[9] H. Brinkmann, C. Kelting, S. Makarov, O. Tsaryova, G. Schnurpfeil, D. Wöhrle, D. Schlettwein, Fluorinated phthalocyanines as molecular semiconductor thin films, phys. stat. sol. (a) 205 (2008) 409-420.

[10] M. Knupfer, H. Peisert, Electronic properties of interfaces between model organic semiconductors and metals, phys. stat. sol. (a) 201 (2004) 10551074 .

[11] J. Wang, H. B. Wang, X. J. Yan, H. C. Huang, D. H. Yan, Organic heterojunction and its application for double channel field-effect transistors, Appl. Phys. Lett. 87 (2005) 093507.

[12] H. Wang, J. Wang, H. Huang, X. Yan, D. Yan, Organic heterojunction with reverse rectifying characteristics and its application in field-effect transistors, Org. Electron. 7 (2006) 369 - 374.

[13] R. Ye, M. Baba, K. Suzuki, K. Mori, Dependence of ambipolar transport on first active layer thickness in organic homostructure transistors, Jpn. J. Appl. Phys. 46 (2007) 2878-2881.

[14] D. Jérome, H. Schulz, Organic conductors and superconductors, Adv. Phys. 31 (1982) 299-490.

[15] Y. Takahashi, T. Hasegawa, Y. Abe, Y. Tokura, G. Saito, Organic metal electrodes for controlled p- and n-type carrier injections in organic fieldeffect transistors, Appl. Phys. Lett. 88 (2006) 073504. 
[16] J.-O. Vogel, I. Salzmann, R. Opitz, S. Duhm, B. Nickel, J. P. Rabe, N. Koch, Sub-nanometer control of the interlayer spacing in thin films of intercalated rodlike conjugated molecules, J. Phys. Chem. B 111 (2007) 14097-14101.

[17] I. Salzmann, S. Duhm, G. Heimel, M. Oehzelt, R. Kniprath, R. L. Johnson, J. P. Rabe, N. Koch, Tuning the ionization energy of organic semiconductor films: The role of intramolecular polar bonds, J. Am. Chem. Soc. 130 (2008) 12870-12871.

[18] A. Opitz, M. Kraus, M. Bronner, J. Wagner, W. Brütting, Bipolar transport in organic field-effect transistors: Organic semiconductor blends versus contact modification, New. J. Phys. 10 (2008) 065006.

[19] N. Mott, R. Gurney, Electronic Processes in Ionic Crystals, Clarendon Press, Oxford, 1940.

[20] P. Murgatroyd, Theory of space-charge limited current enhanced by Frenkel effect, J. Phys. D: Appl. Phys. 3 (1970) 151-156.

[21] E. A. Lucia, F. D. Verderame, Spectra of polycrystalline phthalocyanines in the visible region, J. Chem. Phys. 48 (1968) 2674-2681.

[22] O. Berger, W.-J. Fischer, B. Adolphi, S. Tierbach, V. Melev, J. Schreiber, Studies on phase transformations of Cu-phthalocyanine thin films, J. Mater. Sci. 11 (2000) 331-346.

[23] M. Ashida, N. Uyeda, E. Suito, Unit cell metastable-form constants of various phthalocyanines, B. Chem. Soc. Jpn. 39 (1966) 2616-2624.

[24] A. Hoshino, Y. Takenaka, H. Miyaji, Redetermination of the crystal structure of alpha-copper phthalocyanine grown on $\mathrm{KCl}$, Acta Crystallogr. B 59 (2003) 393-403.

[25] J. O. Ossó, F. Schreiber, V. Kruppa, H. Dosch, M. Garriga, M. I. Alonso, F. Cerdeira, Controlled molecular alignment in phthalocyanine thin films on stepped sapphire surfaces, Adv. Func. Mater. 12 (2002) 455-460.

[26] D. G. de Oteyza, E. Barrena, J. O. Ossó, S. Sellner, H. Dosch, Thicknessdependent structural transitions in fluorinated copper-phthalocyanine ( $F_{16} \mathrm{CuPc}$ ) films, J. Am. Chem. Soc. 128 (2006) 15052-15053.

[27] J. Y. E, S. Kim, E. J. Lim, K. J. Lee, D. J. Cha, B. Friedman, Effects of substrate temperature on copper(II) phthalocyanine thin films, Appl. Surf. Sci. 205 (2003) 274-279.

[28] D. Datta, V. Tripathi, P. Gogoi, S. Banerjee, S. Kumar, Ellipsometric studies on thin film CuPC:C60 blends for solar cell applications, Thin solid films 516 (2008) 7237-7240.

[29] M. I. Alonso, M. Garriga, J. O. Ossó, F. Schreiber, E. Barrena, H. Dosch, Strong optical anisotropies of $\mathrm{F}_{16} \mathrm{CuPc}$ thin films studied by spectroscopic ellipsometry, J. Chem. Phys. 119 (2003) 6335-6340.

[30] W. Brütting, ed., Physics of Organic Semiconductors, Wiley-VCH, Weinheim, 2005

[31] I. Hill, A. Kahn, Z. Soos, R. Pascal, Charge-separation energy in films of $\pi$-conjugated organic molecules, Chem. Phys. Lett. 327 (2000) 181-188.

[32] M. Pope, C. E. Swenberg, Electronic processes in organic crystals and polymers, Oxford university press, New York, 1999.

[33] V. D. Mihailetchi, L. J. A. Koster, J. C. Hummelen, P. W. M. Blom, Photocurrent generation in polymer-fullerene bulk heterojunctions, Phys. Rev. Lett. 93 (2004) 216601.

[34] C. J. Brabec, V. Dyakonov, U. Scherf, Organic Photovoltaics, WileyVCH, Weinheim, 2008

[35] N. S. Sariciftci, L. Smilowitz, A. J. Heeger, F. Wudl, Photoinduced electron-transfer from a conducting polymer to buckminsterfullerene, Science 258 (1992) 1474-1476.

[36] B. Kraabel, D. McBranch, N. S. Sariciftci, D. Moses, A. J. Heeger, Ultrafast spectroscopic studies of photoinduced electron-transfer from semiconducting polymers to C $_{60}$, Phys. Rev. B 50 (1994) 18543-18552.

[37] G. Yu, J. Gao, J. C. Hummelen, F. Wudl, A. J. Heeger, Polymer photovoltaic cells - enhanced efficiencies via a network of internal donoracceptor heterojunctions, Science 270 (1995) 1789-1791.

[38] T. Stübinger, W. Brütting, Photocurrent spectra of bilayers and blends of the organic donor-acceptor system $\mathrm{CuPc} / \mathrm{C}_{60}$, SPIE Proc. 4465 (2002) $102-112$.

[39] J. G. Xue, B. P. Rand, S. Uchida, S. R. Forrest, Mixed donor-acceptor molecular heterojunctions for photovoltaic applications. II. Device performance, J. Appl. Phys. 98 (2005) 124903.

[40] A. Opitz, M. Bronner, J. Wagner, M. Götzenbrugger, W. Brütting, Ambipolar organic semiconductor blends for photovoltaic cells, SPIE Proc. 7002 (2008) 70020J.

[41] K. Lau, J. Tang, H. Sun, C. Lee, S. Lee, D. Yan, Interfacial electronic structure of copper phthalocyanine and copper hexadecafluorophthalo- cyanine studied by photoemission, Appl. Phys. Lett. 88 (2006) 173513.

[42] M. Kröger, S. Hamwi, J. Meyer, T. Dobbertin, T. Riedl, W. Kowalsky, H.$\mathrm{H}$. Johannes, Temperature-independent field-induced charge separation at doped organic/organic interfaces: Experimental modeling of electrical properties, Phys. Rev. B 75 (2007) 235321.

[43] B. Yu, F. Zhu, H. Wang, G. Li, D. Yan, All-organic tunnel junctions as connecting units in tandem organic solar cell, J. Appl. Phys. 104 (2008) 114503 . 